Technological University Dublin

DƯBLIN

ARROW@TU Dublin

2013

\title{
Enhanced Refractive Index Sensor Using a Combination of a Long Period Fiber Grating and a Small Core Singlemode Fiber Structure
}

\author{
Qiang wu \\ Technological University Dublin, qiang.wu@tudublin.ie \\ Hauping Chan \\ City University of Hong Kong \\ Jinhui Yuan \\ Beijing University of Posts and Telecommunications
}

See next page for additional authors

Follow this and additional works at: https://arrow.tudublin.ie/engscheceart

\section{Recommended Citation}

Wu, Q., Chan, H., Yuan, J., Ma, Y., Yang, M., Semenova, Y., Yan, B., Wang, P., Yu, C., and Farrell, G. Enhanced refractive index sensor using a combination of a long period fiber grating and a small core singlemode fiber structure. Measurement Science and Technology, 24 (2013) 094002. doi:10.1088/0957-0233/24/9/ 094002

This Article is brought to you for free and open access by the School of Electrical and Electronic Engineering at ARROW@TU Dublin. It has been accepted for inclusion in Articles by an authorized administrator of ARROW@TU Dublin. For more information, please contact arrow.admin@tudublin.ie, aisling.coyne@tudublin.ie, gerard.connolly@tudublin.ie.

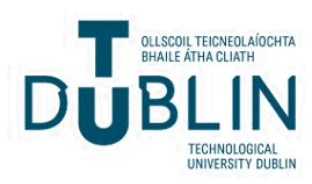




\section{Authors}

Qiang wu, Hauping Chan, Jinhui Yuan, Youqiao Ma, Minwei Yang, Yuliya Semenova, Binbin Yan, Pengfei Wang, Chongxiu Yu, and Gerald Farrell 
Enhanced refractive index sensor using a combination of a long period fiber grating and a small core singlemode fiber structure

This article has been downloaded from IOPscience. Please scroll down to see the full text article.

2013 Meas. Sci. Technol. 24094002

(http://iopscience.iop.org/0957-0233/24/9/094002)

View the table of contents for this issue, or go to the journal homepage for more

Download details:

IP Address: 147.252.236.38

The article was downloaded on 25/07/2013 at 09:49

Please note that terms and conditions apply. 


\title{
Enhanced refractive index sensor using a combination of a long period fiber grating and a small core singlemode fiber structure
}

\author{
Qiang Wu ${ }^{1,4}$, Hai Ping Chan ${ }^{2}$, Jinhui Yuan ${ }^{3}$, Youqiao $\mathrm{Ma}^{1}$, \\ Minwei Yang ${ }^{1}$, Yuliya Semenova ${ }^{1}$, Binbin Yan $^{3}$, Pengfei Wang ${ }^{1}$, \\ Chongxiu Yu $^{3}$ and Gerald Farrell ${ }^{1}$ \\ ${ }^{1}$ Photonics Research Center, School of Electronic and Communications Engineering, \\ Dublin Institute of Technology, Kevin Street, Dublin 8, Ireland \\ ${ }^{2}$ Department of Electronic Engineering, City University of Hong Kong, Hong Kong, \\ People's Republic of China \\ ${ }^{3}$ Laboratory of Information Photonics and Optical Communications, Beijing University of Posts and \\ Telecommunications, Ministry of Education, PO Box 72 (BUPT), Beijing, People's Republic of China \\ E-mail: qiang.wu@dit.ie
}

Received 9 October 2012, in final form 28 December 2012

Published 24 July 2013

Online at stacks.iop.org/MST/24/094002

\begin{abstract}
An enhanced refractive index (RI) sensor based on a combination of a long period fiber grating (LPG) and a small core singlemode fiber (SCSMF) structure is proposed and developed. Since the LPG and SCSMF transmission spectra experience a blue and a red shift respectively as the surrounding RI (SRI) increases, the sensitivity is improved by measuring the separation between the resonant wavelengths of the LPG and SCSMF structures. Experimental results show that the sensor has a sensitivity of $1028 \mathrm{~nm} /$ SRI unit in the SRI range from 1.422 to 1.429 , which is higher than individual sensitivities of either structure alone used in the experiment. Experimental results agree well with simulation results.
\end{abstract}

Keywords: refractive index sensor, long period fiber grating

(Some figures may appear in colour only in the online journal)

\section{Introduction}

Optical fiber based refractive index (RI) sensors have attracted wide interest due to their unique advantages such as immunity to electromagnetic interference, small size, high sensitivity, remote operation capability, etc. The techniques used to implement fiber based RI sensing include a fiber Bragg grating (FBG) [1], long period fiber grating (LPG) [2, 3], surface plasmon resonance [4], tapered fiber [5] and a singlemodemultimode-singlemode (SMS) fiber structure [6, 7]. Among these techniques, an SMS fiber structure based optical sensor has the advantages of low cost and ease of fabrication and

\footnotetext{
${ }^{4}$ Author to whom any correspondence should be addressed.
}

hence has attracted wide research interest [6-13]. As for RI sensing, our recent investigations have shown that an etched SMS fiber structure has high sensitivity with a demonstrated maximum sensitivity of $1815 \mathrm{~nm} / \mathrm{RIU}$ [6]. An alternative approach is to use a small core singlemode fiber (SCSMF) as a substitute for the etched MMF to construct an SCSMF structure based refractometer [14, 15]. The advantage of using an SCSMF is that it can be manufactured without the use of fiber etching, which means it is possible to guarantee the accuracy of the fiber diameter and smoothness of the fiber surface, yielding a simpler and more repeatable SCSMF structure based refractometer. Our investigations have shown that this sensor exhibits wavelength red-shift as the surrounding RI (SRI) increases [15]. On the other hand, the 


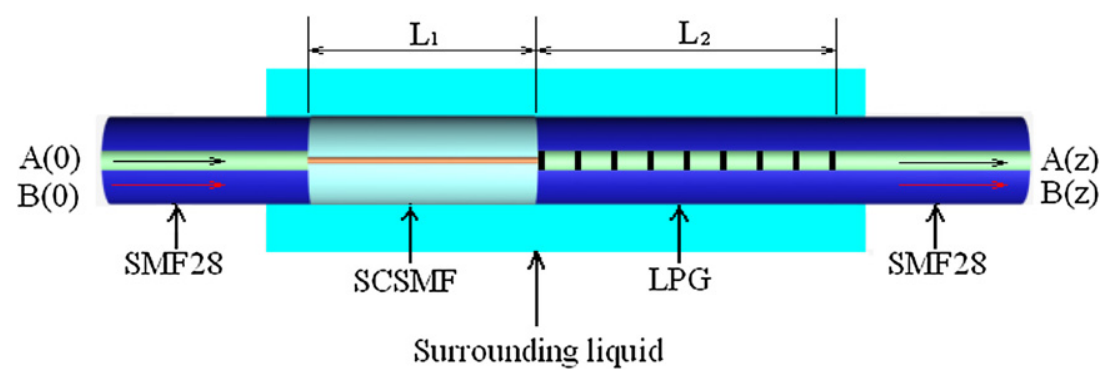

Figure 1. Schematic diagram of an LPG and SCSMF structure based RI sensor.

LPG is a promising technique for optical sensing [16, 17], for example an LPG can be used as an RI sensor and may exhibit wavelength blue-shift as the RI increases. If the two types of sensors are combined with each other, the sensitivity will be improved by monitoring the separation wavelength shifts between the resonant wavelengths of the LPG and SCSMF structures. In this paper we investigate for the first time the use of a combination of LPG and SCSMF fiber structures for RI sensing and show that the sensitivity achievable is higher than that of either of the structures alone.

\section{Principle}

A schematic diagram of a combined LPG and SCSMF structure based RI sensor is shown in figure 1. Both the SCSMF section and SMF28 in figure 1 typically have a step index profile. Cladding modes will be excited in the SCSMF section when the light enters from the input SMF28. As light is transmitted through the SCSMF, interference takes place between these cladding modes. These cladding modes will also be influenced by the SRI. At the output of the SCSMF section, the power of the core mode coupled to the output SMF28 is found to be wavelength dependent. Furthermore as an LPG is imprinted in the output SMF28 fiber, the power in this core mode is coupled to a cladding mode by the LPG. The resonant wavelength of the LPG will also be influenced by the SRI. Therefore as the RI of the surrounding liquid changes, the resonant wavelengths of both the LPG and SCSMF structures change, but with different wavelength shift directions: for example, if the SRI decreases, the SCSMF structure exhibits blue shift, while the LPG will have red shift. This separation wavelength shift is larger than the individual wavelength shifts of either LPG or SCSMF structure alone, and hence the detectable sensitivity to SRI changes is higher than that of either of the individual structures alone. Moreover, since the LPG and SCSMF structures display different temperature responses, it may be possible with the combined structure to measure both temperature and RI.

Assuming the light in the input SMF28 has a fundamental mode field distribution $A(r, 0)$ within the core area and the field profile within an SCSMF is $\Psi(r)$, the input field at the SCSMF can be written as [15]

$$
\Psi(r)=\sum_{m=1}^{M} \Psi_{m}(r),
$$

$$
A(r, 0)=\sum_{m=1}^{M} b_{m} \Psi_{m}(r),
$$

where $A(r, 0)$ is the eigenmode of the SMF28, $\Psi_{m}(r)$ is the $m$ th eigenmode of the step index optical fiber SCSMF, $M$ is the total number of modes supported in the SCSMF and $b_{m}$ is the excitation coefficient for the $m$ th mode, which can be expressed as

$$
b_{m}=\frac{\int_{0}^{\infty} A(r, 0) \Psi_{m}(r) r \mathrm{~d} r}{\int_{0}^{\infty} A(r, 0) A(r, 0) r \mathrm{~d} r} .
$$

The field within the SCSMF section at a propagation distance $L_{1}$ can thus be calculated by

$$
E\left(r, L_{1}\right)=\sum_{m=1}^{M} b_{m} \Psi_{m}(r) \exp \left(\mathrm{j} \beta_{m} L_{1}\right),
$$

where $\beta_{m}$ is the propagation constant of the $m$ th eigenmode within the SCSMF. At the interface between the SCSMF and the output SMF28, the light will be coupled to both core and cladding modes within SMF28, which can be expressed as

$$
\begin{aligned}
& A\left(L_{1}\right)=\frac{\int_{0}^{\infty} E\left(r, L_{1}\right) A(r, 0) r \mathrm{~d} r}{\int_{0}^{\infty} A(r, 0) A(r, 0) r \mathrm{~d} r}, \\
& B_{n}\left(L_{1}\right)=\frac{\int_{0}^{\infty} E\left(r, L_{1}\right) \Phi_{n}(r) r \mathrm{~d} r}{\int_{0}^{\infty} \Phi_{n}(r) \Phi_{n}(r) r \mathrm{~d} r},
\end{aligned}
$$

where $\Phi_{n}(r)$ is the $n$th cladding mode within the SMF28.

It is noted that in the above equations, the mode fields are normalized as

$$
\begin{aligned}
\int_{0}^{\infty} A(r, 0) A(r, 0) r \mathrm{~d} r & =\int_{0}^{\infty} \Psi_{m}(r) \Psi_{m}(r) r \mathrm{~d} r \\
& =\int_{0}^{\infty} \Phi_{n}(r) \Phi_{n}(r) r \mathrm{~d} r=1 .
\end{aligned}
$$

As both the core and cladding modes propagate through the LPG, the output from the LPG can be expressed as [18]

$$
\begin{aligned}
& \left(\begin{array}{l}
A\left(L_{1}+L_{2}\right) \\
B\left(L_{1}+L_{2}\right)
\end{array}\right) \\
& \quad=\left(\begin{array}{ll}
\cos \left(\gamma L_{2}\right)+\mathrm{i} \frac{\sigma}{\gamma} \sin \left(\gamma L_{2}\right) & \mathrm{i} \frac{\kappa}{\gamma} \sin \left(\gamma L_{2}\right) \\
\mathrm{i} \frac{\kappa}{\gamma} \sin \left(\gamma L_{2}\right) & \cos \left(\gamma L_{2}\right)-\mathrm{i} \frac{\sigma}{\gamma} \sin \left(\gamma L_{2}\right)
\end{array}\right) \\
& \quad \times\left(\begin{array}{c}
A\left(L_{1}\right) \\
B_{n}\left(L_{1}\right)
\end{array}\right),
\end{aligned}
$$

where $\kappa$ is the 'ac' cross-coupling coefficient, $\sigma$ is a general 'dc' self-coupling coefficient and $\gamma=\sqrt{\kappa^{2}+\sigma^{2}}$. 



Figure 2. Simulated $(a)$ spectral response and $(b)$ wavelength shift of the sensor with different SRI.

\section{Numerical simulations}

Simulations based on the above structure were carried out as shown in figure 2 demonstrating the spectral response under different SRI conditions. In this simulation the SCSMF has core and cladding diameters of 2.2 and $125 \mu \mathrm{m}$ respectively and the refractive indices of the core and cladding are 1.451 and 1.445 respectively, the SMF28 has core and cladding diameters of 8.3 and $125 \mu \mathrm{m}$ and the refractive indices of the core and cladding are 1.449 and 1.444 respectively, and the LPG has a period of $390 \mu \mathrm{m}$ and length of $15.6 \mathrm{~mm}$ (40 periods) which correspond to the $\mathrm{LP}_{06}$ cladding mode.

Figure 2(a) shows that as the SRI increases, the LPG experiences wavelength blue-shift but the SCSMF structure undergoes wavelength red-shift monotonically. Figure $2(b)$ shows the simulated wavelength shifts for both the LPG and SCSMF structure wavelength dips. From figure $2(b)$ it is obvious that both LPG and SCSMF have a nonlinear response for wavelength shift versus SRI; the higher the SRI range, the higher the sensitivity for both resonant wavelengths of LPG and SCSMF. The estimated sensitivities for the LPG and SCSMF dips are -40 and $100 \mathrm{~nm} /$ SRIU (SRI unit) in the SRI range from 1.33 to 1.34 , and -280 and $820 \mathrm{~nm} / \mathrm{SRIU}$ in the range from 1.41 to 1.42 respectively. When the separation wavelength shift is considered, the sensitivity increases to 140 and $1100 \mathrm{~nm} / \mathrm{SRI}$ in the SRI range from 1.33 to 1.34 and from 1.41 to 1.42 respectively, which is the sum of the individual LPG and SCSMF sensitivities.

\section{Experimental investigation}

An experimental setup to utilize the combined LPG and SCSMF structure for RI sensing is shown in figure 3. The SCSMF used in our experiments was 460HP which has a step index profile. The LPG was fabricated by a $\mathrm{CO}_{2}$ laser in an SMF28 with a grating period of $390 \mu \mathrm{m}$ and length of $15.6 \mathrm{~mm}$. One end of the SCSMF is fusion spliced to an SMF28 and the other end of the SCSMF is fusion spliced to an LPG. The length of the SCSMF is $20 \mathrm{~mm}$. The combined RI sensor structure is driven by a broadband source operating in the infrared wavelength range and the spectrum was measured

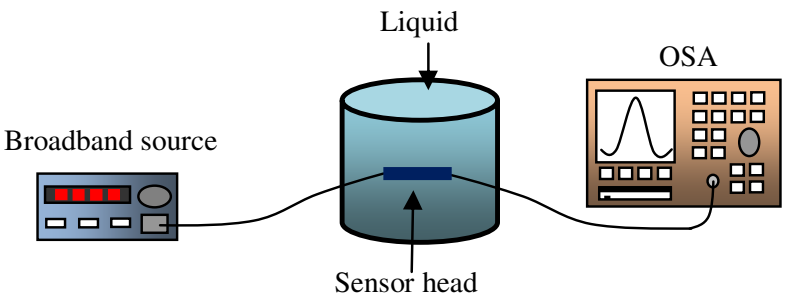

Figure 3. Schematic experimental setup for RI sensing.

by an optical spectrum analyzer (OSA) (Agilent 86142B) with a resolution of $0.5 \mathrm{~nm}$.

The measured spectral response of the combined LPG and SCSMF structure immersed in different RI liquids is shown in figure 4(a), displaying as expected two distinct dip or resonant regions. The RI liquids were made with dimethyl sulfoxide mixed with water in different concentrations. The refractive indices of the mixed liquids were then calibrated with an Abbe 5 refractometer at a wavelength of $589 \mathrm{~nm}$.

Figure 4(a) shows that as the SRI increases, the resonant wavelength of the LPG decreases and the resonant wavelength of the SCSMF structure increases monotonically. Figure 4(b) shows the measured wavelength shifts for both the LPG and SCSMF structure wavelength dips.

From figure $4(b)$ it is estimated that the LPG and SCSMF dips have sensitivities of -38 and $59 \mathrm{~nm} / \mathrm{SRIU}$ in the SRI range from 1.335 to 1.36 , and -319 and $709 \mathrm{~nm} / \mathrm{SRIU}$ in the range from 1.422 to 1.429 respectively. When the separation wavelength shift is considered, the sensitivity increases to 97 and $1028 \mathrm{~nm} / \mathrm{SRI}$ in the SRI range from 1.335 to 1.36 and from 1.422 to 1.429 respectively, which is the sum of the individual LPG and SCSMF sensitivities. This sensitivity is much higher than that of an FBG based RI sensor, for example a sensitivity of $2.3 \mathrm{~nm} / \mathrm{RIU}$ in an RI range from 1.446 to 1.458 is reported in [1].

The temperature dependence of the RI sensor was also experimentally investigated as shown in figure 5. Since the test RI liquids used in the experiments utilized different mixtures of dimethyl sulfoxide and water with different concentrations, the dimethyl sulfoxide will evaporate as the temperature increases which in turn will change the RI of the liquids. Thus 



Figure 4. Measured $(a)$ spectral response and $(b)$ wavelength shifts of the sensor with different SRI.



Figure 5. Wavelength shift versus temperature of the RI sensor immersed in pure water.

investigations on the temperature dependence of the RI sensor were only carried out by immersing the sensor in pure water, the temperature of which could be controlled.

Figure 5 shows the sensor response, for the sensor immersed in pure water, as the temperature increases from 25 to $45{ }^{\circ} \mathrm{C}$. Both the LPG and SCSMF experience wavelength shifts in the same direction with value of -0.125 and $-0.427 \mathrm{~nm}^{\circ} \mathrm{C}^{-1}$ respectively. Since the LPG and SCSMF have opposite wavelength shift directions as SRI varies, it is possible to set up two independent equations which are functions of the LPG and SCSMF wavelength shifts versus SRI and temperature, and hence by solving the two equations it is possible to simultaneously determine both temperature and RI.

\section{Conclusions}

In this paper we propose to use a combined LPG and SCSMF structure as an enhanced sensitivity RI sensor. The underlying principle is that the transmission spectra of both the LPG and SCSMF structures are sensitive to the SRI but with different wavelength shift directions for the same SRI change. By measuring the wavelength separation shift of the LPG and SCSMF structure, the sensitivity can be improved and both our theoretical and experimental investigations have demonstrated that this sensor has a higher sensitivity than any individual sensor based on either an LPG or SCSMF structure alone.

\section{Acknowledgments}

This work was supported by Science Foundation Ireland under grant no 07/SK/I1200, 11/TIDA/B2051, 07/SK/I1200STTF11; Open Fund of State Key Laboratory of Information Photonics and Optical Communications (Beijing University of Posts and Telecommunications), People's Republic of China; Dublin Institute of Technology under the Fiosraigh Research Scholarship; Irish Research Council for Science, Engineering and Technology; and Marie-Curie Actions under FP7.

\section{References}

[1] Han M, Guo F W and Lu Y F 2010 Optical fiber refractometer based on cladding-mode Bragg grating Opt. Lett. 35 399-401

[2] Tsuda H and Urabe K 2009 Characterization of long-period grating refractive index sensors and their applications Sensors 9 4559-71

[3] Korposh S, Lee S W, James S W and Tatam R P 2011 Refractive index sensitivity of fibre-optic long period gratings coated with $\mathrm{SiO}_{2}$ nanoparticle mesoporous thin films Meas. Sci. Technol. 22075208

[4] Lee J Y and Tsai S K 2011 Measurement of refractive index variation of liquids by surface plasmon resonance and wavelength-modulated heterodyne interferometry $\mathrm{Opt}$. Commun. 284 925-9

[5] Tai Y H and Wei P K 2010 Sensitive liquid refractive index sensors using tapered optical fiber tips Opt. Lett. 35 944-6

[6] Wu Q, Semenova Y, Wang P and Farrell G 2011 High sensitivity SMS fiber structure based refractometer-analysis and experiment Opt. Express $197937-44$

[7] Yang L, Xue L L, Che D and Qian J R 2012 Guided-mode-leaky-mode-guided-mode fiber structure and its application to high refractive index sensing Opt. Lett. 37 587-9 
[8] Li L C, Xia L, Xie Z H and Liu D M 2012 All-fiber Mach-Zehnder interferometers for sensing applications Opt. Express 20 11109-20

[9] Wu Q, Semenova Y, Yan B B, Ma Y Q, Wang P, Yu C X and Farrell G 2011 Fiber refractometer based on a fiber Bragg grating and single-mode-multimode-single-mode fiber structure Opt. Lett. 36 2197-9

[10] Tripathi S M et al 2009 Strain and temperature sensing characteristics of single-mode-multimode-single-mode structures J. Lightwave Technol. 27 2348-56

[11] Wu Q, Semenova Y, Mathew J, Wang P and Farrell G 2011 Humidity sensor based on a singlemode hetero-core fiber structure Opt. Lett. 36 1752-4

[12] Soldano L B and Pennings E C M 1995 Optical multi-mode interference devices based on self-imaging: principles and applications J. Lightwave Technol. 13 615-27

[13] Wu Q, Semenova Y, Wang P and Farrell G 2011 Fibre heterostructure for simultaneous strain and temperature measurement Electron. Lett. 47 713-4
[14] Xia T H, Zhang A P, Gu B B and Zhu J J 2010 Fiber-optic refractive-index sensors based on transmissive and reflective thin-core fiber modal interferometers $O p t$. Commun. $2832136-9$

[15] Wu Q, Semenova Y, Wang P and Farrell G 2011 A comprehensive analysis verified by experiment of a refractometer based on an SMF28-small-core singlemode fiber (SCSMF)-SMF28 fiber structure J. Opt. 13125401

[16] Frazao O, Falate R, Fabris J L, Santos J L, Ferreira L A and Araujo F M 2006 Optical inclinometer based on a single long-period fiber grating combined with a fused taper Opt. Lett. 31 2960-2

[17] Frazao O, Viegas J, Caldas P, Santos J L, Araujo F M, Ferreira L A and Farahi F 2007 All-fiber Mach-Zehnder curvature sensor based on multimode interference combined with a long-period grating Opt. Lett. 32 3074-6

[18] Erdogan T 1997 Fiber grating spectra J. Lightwave Technol. 15 1277-94 